\title{
Exploiting Senescence for Cancer Treatment
}

\section{Mayur Arvind Gadhikar ${ }^{1}$ and Pankaj Kumar Singh ${ }^{2 *}$}

${ }^{1}$ Department of Head and Neck Surgery, University of Texas, MD Anderson Cancer Center, 1515 Holcombe Blvd, Houston Texas, 77030, USA

${ }^{2}$ Department of Experimental Radiation Oncology, University of Texas, MD Anderson Cancer Center, 1515 Holcombe Blvd, Houston Texas, 77030 , USA

\begin{abstract}
Senescence is considered as a cellular defensive response evoked by diverse stimuli to combat tumor development and progression. An evolving body of knowledge indicates that tumor cells are capable of undergoing senescence when latent senescence pathways are reengaged. Given the apparent benefits associated with senescence induction as a therapy outcome, targeting of senescence triggering factors is being actively pursued. In this review, we briefly describe modes of senescence induction and discuss therapy approaches being undertaken for the development of prosenescence anti-cancer therapies.
\end{abstract}

Keywords: Senescence; Cancer therapy; Induction of senescence

\section{Introduction}

Replicative senescence was first described by Hayflick and Moorehead about 40 years ago, when they observed that in vitro serial passages of normal human fibroblasts culminates in an irreversible exit from the cell cycle [1,2]. Later conflicting scientific data and arguments surfaced which prompted the scientific experts to refine the original definition of senescence. Cellular senescence is now defined as an irreversible non-proliferative state of cells, characterized by the permanent exit from cell division cycle and non-responsiveness to mitogenic signaling and oncogenic influence [3]. It is important to note that senescent cells remain viable and metabolically active despite undergoing stable proliferation arrest. In addition, senescent cells secrete a multitude of protein factors with diverse physiological function. These cellular secretions are collectively termed as senescenceassociated secretory phenotype (SASP) $[4,5]$.

\section{Replicative senescence}

Two critical pathways influence the onset of replicative senescence. The first is the DNA Damage Response (DDR) pathway that is triggered upon the exposure of telomeric ends and on attrition of telomeres [6]. In the DDR pathway, Ataxia telangiectasia mutated (ATM), Ataxia telangiectasia and Rad3 related (ATR), Checkpoint kinase 1 (Chk1), and Checkpoint kinase 2 (Chk2) are the main mediators that phosphorylate and activate many proteins including p53 which promote the cellular exit from the cell proliferation cycle [6-9]. It is intriguing to note that in many cancers sustained DDR signaling is observed yet the cells do not manifest senescence phenotype [10]. The second pathway operates via de-repression of cyclin-dependent kinase Inhibitor $2 \mathrm{~A}$ (CDKN2A) locus which encodes $\mathrm{p} 16$ and ARF. p16 protein inhibits G1 phase cyclin dependent kinases, Cdk4 and Cdk6, while ARF regulates p53 through inactivation of its negative regulator, Mouse double minute 2 homolog (MDM2). The molecular mechanisms underlying the de-repression of CDKN2A locus are not completely known, however, it is wellrecognized that disengagement of polycomb repression complexes from the CDKN2A locus is critically involved in this process $[6,11]$. The extent to which these two individual pathways are engaged for senescence induction is greatly influenced by the organism of origin, type of cell and the nature of stress $[6,11]$.

\section{Oncogene induced senescence (OIS)}

OIS was first seen in normal fibroblasts as an in-vitro cellular response to forced overexpression of oncogenic RAS (G12V) with ensuing phenotype very similar to replicative senescence [12]. Studies demonstrated that this phenotype was a result of DNA hyperreplication which triggered the activation of $S$ phase-specific DDR.
Despite the differences in the mechanism of initiation of DDR, OIS and replicative senescence employ similar primary pathways and effectors of DDR signaling. In congruence with this notion, it is found that the OIS response fails to set in the cells with deficient ATM activity or when the DNA damage sensing machinery is inactivated or DDR signaling to p53 is blocked.

The presence OIS was detected in various human and mouse premalignant lesions by many independent groups, however, their advanced tumors lacked OIS features [13-16]. Examples of premalignant lesions in which OIS was observed include nevi, benign precursor lesions to melanoma, early stage lung and pancreatic cancer, serrate colon cancer models, 7,12-dimethylbenz(a) anthracene (DMBA) and Tissue plasminogen activator (TPA) induced skin papilloma $[14,17,18]$. Furthermore, inactivation of tumor suppressor genes was also seen to evoke senescence phenotype. For example, loss of Phosphatase and tensin homolog (PTEN) in mouse prostate, Nuclear Respiratory Factor 1 (NRF1) loss in neurofibromas, von Hippel-Lindau (VHL) loss in renal cancer and $\mathrm{Rb}$ in thyroid cancers all resulted in premalignant lesions with senescence markers [6,19-21]. There is now abundant evidence to support that oncogenic activation spark the appearance of premalignant lesions but also could alongside initiate senescence program. Abrogation of this senescence program is essential for progression of tumor (Figure 1). For instance, development of melanomas can be strikingly accelerated upon inactivation of p53 or p16 in nevi lesions induced by oncogenic BRAF expression [6]. Similarly, p53 ablation in PTEN (-/-) prostate neoplastic lesions can accelerate the development of advanced metastatic prostate cancer [22]. It is clear from these examples that if mutations deactivating senescence program occur, then it could lead to the reversal of OIS and promote tumor progression (Figure 1). A corollary to this inference is that senescence triggered in the paraneoplastic lesions serves as an effective barrier against tumorigenesis and that it needs to be breached for tumor progression.

${ }^{*}$ Corresponding author: Dr. Pankaj Kumar Singh, Professor, University of Texas, Anderson Cancer Center, US, Tel: 001 713-792-2121, E-mail: PKSingh@mdanderson.org

Received September 07, 2016; Accepted September 08, 2016; Published September 15, 2016

Citation: Gadhikar MA, Singh PK (2016) Exploiting Senescence for Cance Treatment. Mol Biol 5: 172. doi:10.4172/2168-9547.1000172

Copyright: (c) 2016 Gadhikar MA, et al. This is an open-access article distributed under the terms of the Creative Commons Attribution License, which permits unrestricted use, distribution, and reproduction in any medium, provided the original author and source are credited. 


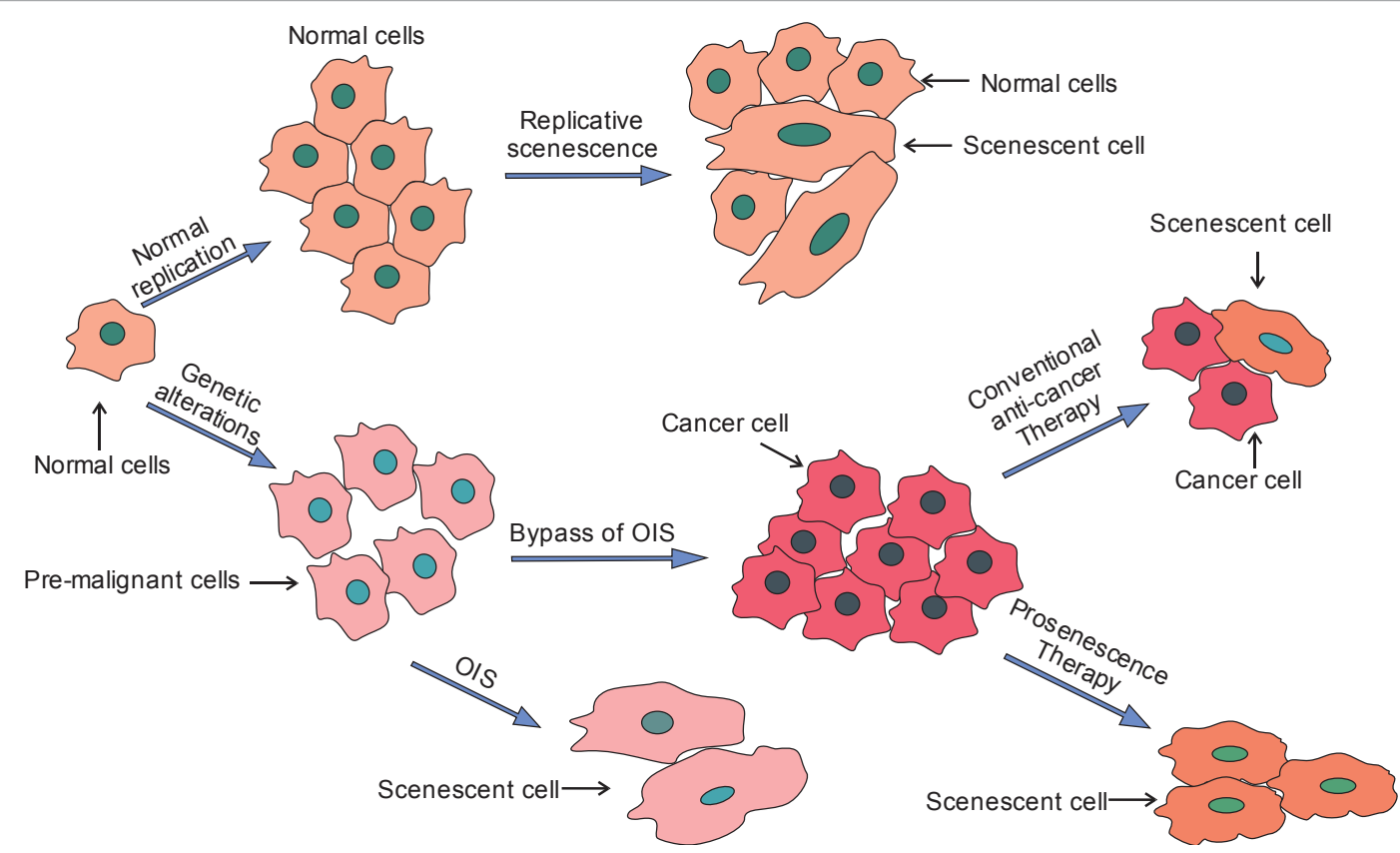

Figure 1: Senescence role in tumorigenesis and therapy induced senescence. Genetic alterations in normal cells result in abnormal proliferation leading to premalignant lesions. Alongside, built-in anti-tumor mechanisms such as OIS are triggered. Acquisition of further tumor promoting mutations leads to bypass of OIS and development of cancer. Treatments with conventional therapies induce senescence in a subset of malignant cells. Presently, therapies directed at specifically inducing senescence are being investigated for their anti-tumor potential.

\section{Therapy induced senescence (TIS)}

Senescence induction as a form of therapy outcome has achieved active clinical interest due to the observations that some tumors cells are capable of undergoing senescence in response to genotoxic treatments. These therapy outcomes indicate that senescence program is intact, nevertheless latent inside many tumor cells and that therapies aimed at reviving senescence may unleash pathways critical to senescence induction. p16INK4a and p53 tumor suppressor pathways are critical to the induction of therapy induced senescence (TIS). Inactivation of either of these two pathways confers resistance to cancer treatment which suggests that ability to actuate a strong senescence response governs the therapy outcome in cancer cells. It has also been reported that the dose strength of genotoxic therapy influences the choice of cellular response pathways in cancer cells. For example, we have shown that supraphysiological dose of cisplatin leads to apoptosis in head and neck squamous cell carcinoma (HNSCC) cells regardless of p53 status; however, a physiological cisplatin dose leads to robust senescence response in wild type p53 HNSCC cells [23]. Advanced malignancies frequently harbor epigenetic silencing, losses, or mutations in the genes critical for TIS pathway induction. Indeed, it is not surprising to see that conventional therapy resistances often arise from the same genetic alterations critical for tumor progression.

\section{Therapy approaches for senescence induction}

Due to the recognized complications arising out of untoward side effects, the genotoxic therapies for cancer management are utilized less enthusiastically. Drug therapies designed to selectively induce senescence in cancer cells, i.e., prosenescence therapies, have been proposed as a novel strategy for cancer treatment. In line with this approach, several drugs therapies positively influencing senescence induction or targeting pathways operating antithetically to senescence program have emerged recently (Figure 2). We seek to present a brief overview of these therapy approaches and the rationale behind their application.

\section{Reengagement of p53 tumor suppressor networks}

Incipient tumors frequently inactivate p16/Rb and p53 tumor suppressive networks to escape OIS and progress into malignancy. Therefore, reengagement of these tumor suppressor networks could reinstate senescence program and arrest tumor growth [9]. While this strategy may be ineffective in tumor harboring p53 deletions, therapeutics augmenting 53 function via MDM2 inhibition or those capable of restoring normal p53 function in p53 mutant tumors could be beneficial [24-27]. Evidences supporting this approach have come from mouse models of lymphoma, sarcoma and hepatocellular carcinoma [6]. In lymphoma, restoration of p53 activity lead to induction of apoptosis and tumor regression, while in sarcomas senescent features with cell cycle arrest were grossly evident [28]. Oncogenic HRASG12V driven HCC exhibited senescent features with negligible apoptosis when p53 was reactivated [29]. Examples of investigational drugs operating through the mechanism of action involving p 53 modulation include Nutlin and PRIMA1 [24,26,27]. Recently, COTI-2, a novel small molecule inhibitor with purported p53 based mechanism of action has entered phase I clinical trial [30].

\section{Targeting cyclin dependent kinase activity}

Cyclin dependent kinase (CDK) inhibitors can promote selective prosenescence response in tumor cells harboring appropriate genetic alterations. For example, c-myc driven tumor cells underwent senescence after inhibition of Cdk2 [31]. Likewise, genetic targeting of cdk4 in oncogenic K-RASG12V driven lung adenomas resulted in induction of senescence and tumor regression [32]. A similar senescent phenotype was observed in advanced lung adenocarcinomas upon cdk4 inactivation. Induction of senescence and suppression of tumorigenesis was also observed upon treatment with skp2 inhibitor MLN4924 in mouse models of various cancers that harbored PTEN loss [33]. 


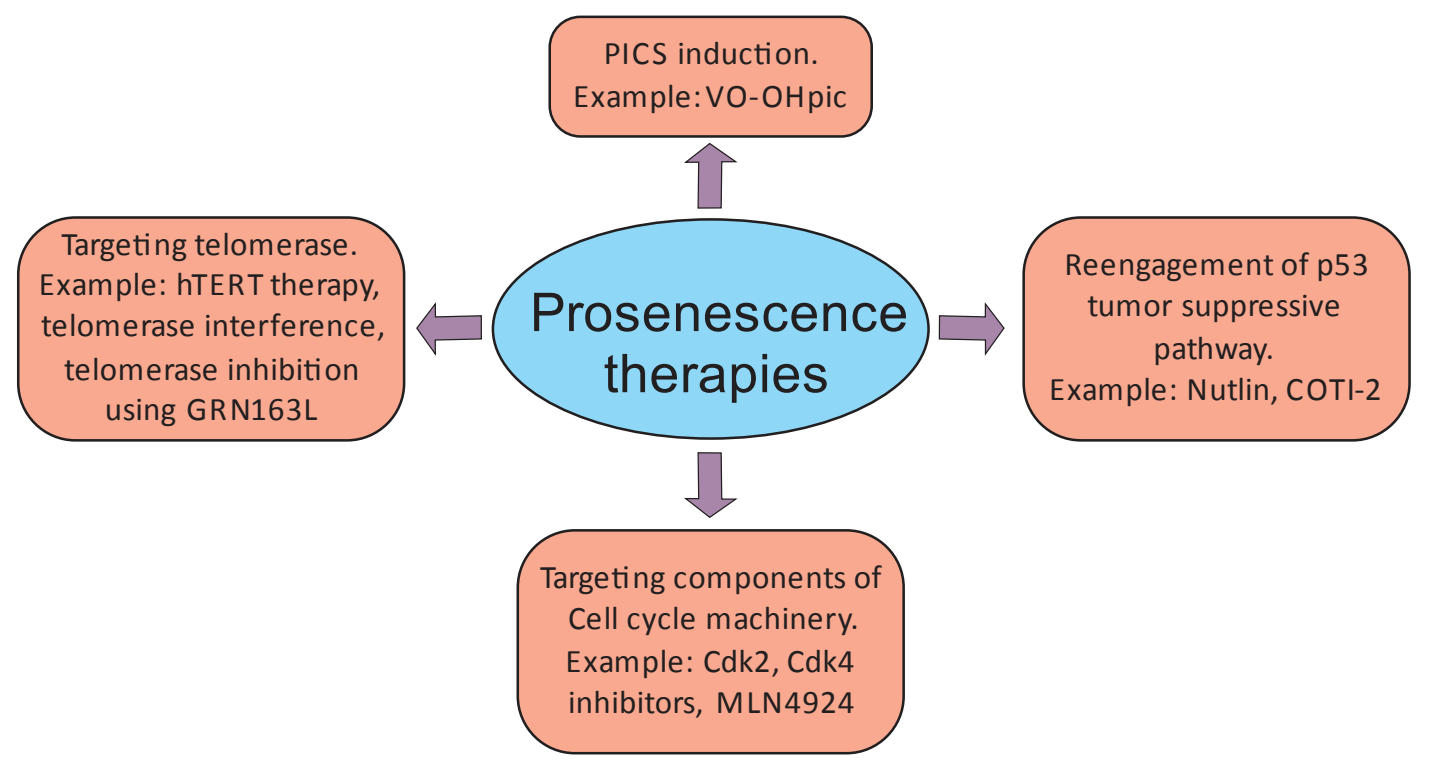

Figure 2: Prosenescence therapy approaches for cancer intervention are 1) reengagement of p53 tumor suppressive pathway 2) targeting components of cell cycle machinery 3) targeting telomerase 4) Induction of PICS response.

\section{Targeting telomerase function}

Around $90 \%$ of tumors acquire telomerase activity to overcome the replicative barrier imposed due to eroded telomeres. Conforming to these findings, genetically engineered mouse models deficient in telomerase were found less susceptible to cancer [6]. Unsurprisingly, therapies aimed at inhibiting telomerase activity are now being evaluated for their anticancer potential. A host of therapeutic strategies targeting telomerase are currently under active investigation. These include direct enzymatic inhibition of telomerase, hTERT therapy, telomerase interference, telomere locking or stabilizing approaches, and telomerase vaccines [34,35]. Among the different drugs developed based on these approaches, GRN163L, a telomerase inhibitor, with promising anti-tumor activity has progressed into later stages of clinical trial [9]. It remains to be seen to what extent senescence would contribute to these therapy outcomes as gross aneuploidies and apoptosis are frequently reported consequences of telomerase inhibition [6].

\section{Inducers of PICS response}

While it is counterintuitive to think that inhibition of tumorsuppressor could have any beneficial outcomes, VO-OHpic based inhibition of PTEN in hemizygous PTEN tumors (+/-) resulted in senescence response solely in PTEN $-/+$ cells with no disastrous consequence on the surrounding homozygous PTEN $+/+$ cells. This senescence response was labelled as PTEN loss Induced Senescence (PICS) [6,9]. Augmented PTEN levels or inhibition of PI3K can also give rise to senescence, nevertheless, slight changes in opposite direction can elicit undesirable effects suggesting that PTEN/PI3K pathway are tightly regulated in cells. These findings highlight that application of prosenescence therapies should be undertaken judiciously with proper regard to the genetic context.

\section{Senescence secretome - an untapped avenue for prosenescence therapy development}

Although permanently non-proliferative, senescent cells manage to influence their surrounding through the release of soluble factors and extracellular proteins. These secreted factors termed as SASP or senescence secretome was initially investigated for protumorigenic properties, however, their role in tumor suppression have also come to light $[7,8]$.The senescence secretome exerts tumor suppressive function through stable maintenance of cell cycle arrest and signaling to and engaging the immune system. Examples of secretome components supporting senescence phenotype include IL-6, IL- 8 and TGF- $\beta[4,6,9]$. Thus, approaches aimed at modulating components of senescence secretome to reinforce senescence phenotype may offer therapeutic benefit. However, it is worthy to note that the same components may discharge protumorigenic actions in a different context. For instance, both IL6 and IL8 display protumorigenic function in tumor models driven by oncogenic RAS [4]. It is unclear what determines this reversal from tumor suppressive function to protumorigenic, but the presence of $\mathrm{p} 16 / \mathrm{Rb}$ and $\mathrm{p} 53$ pathways are thought to be involved $[4,6]$. Although we have been equipped with considerable knowledge on senescence secretome and bear collection of reagents of small molecules and antibodies targeting SASP components, no study as yet has sought to evaluate the utility of prosenescence therapies targeting senescent secretome. We hope that future research in this area of investigation will give us insight on the utility of such approaches.

\section{Conclusion}

Accumulating evidences have highlighted the significance of senescence as an initial physiological response to tumor development. The observations of senescence induction in various cellular models after treatment with current conventional therapies suggest that therapeutic benefits imparted due to senescence have been incompletely harnessed. Nevertheless, such exploration may be viewed with skepticism because senescence as an anti-tumor therapy results in permanent exit from cell cycle whereas other outcomes such as apoptosis or necrosis ensure complete elimination of affected cells. However, examples of senescent cell elimination through immune system recruitment by secretome network indicate that prosenescence therapies may offer two-fold advantage, by inducing irreversible proliferation arrest and engagement of immune system to clear off premalignant as well as malignant cells. We envisage that a detailed mapping of molecular pathways directing 
senescence and identification of senescence regulators by screening experiments from in vitro cellular models may be critical to develop novel prosenescence therapies. Given the state of current knowledge, we predict that constituents of senescent secretome and drugs aimed at modulating epigenetic regulators of senescence could furnish opportunities for development of prosenescence therapies.

\section{References}

1. Saab R (2010) Cellular senescence: many roads, one final destination. ScientificWorld Journal 10: 727-741.

2. Hayflick L, Moorhead PS (1961) The serial cultivation of human diploid cell strains. Exp Cell Res 25: 585-621.

3. Sharpless NE, Sherr CJ (2015) Forging a signature of in vivo senescence. Nat Rev Cancer 15: 397-408.

4. Kuilman T, Peeper DS (2009) Senescence-messaging secretome: SMS-ing cellular stress. Nat Rev Cancer 9: 81-94.

5. Coppe JP (2010) The senescence-associated secretory phenotype: The dark side of tumor suppression. Annu Rev Pathol 5: 99-118.

6. Acosta JC, Gil J (2012) Senescence: A new weapon for cancer therapy. Trends Cell Biol 22: 211-219.

7. Salama R (2014) Cellular senescence and its effector programs. Genes Dev 28: 99-114.

8. Kuilman T (2010) The essence of senescence. Genes Dev 24: 2463-2479.

9. Nardella C (2011) Pro-senescence therapy for cancer treatment. Nat Rev Cancer 11: 503-511.

10. Frederick MJ (2011) Phosphoproteomic analysis of signaling pathways in head and neck squamous cell carcinoma patient samples. Am J Pathol 178: 548-571.

11. Munoz-Espin D, Serrano M (2014) Cellular senescence: from physiology to pathology. Nat Rev Mol Cell Biol 15:482-496.

12. Serrano M (1997) Oncogenic ras provokes premature cell senescence associated with accumulation of p53 and p16INK4a. Cell 88:593-602.

13. Braig M (2005) Oncogene-induced senescence as an initial barrier in lymphoma development. Nature 436: 660-665.

14. Collado M (2005) Tumour biology: Senescence in premalignant tumours Nature 436: 642

15. Lazzerini Denchi E (2005) Deregulated E2F activity induces hyperplasia and senescence-like features in the mouse pituitary gland. Mol Cell Biol 25: 2660-2672

16. Michaloglou C (2005) BRAFE600-associated senescence-like cell cycle arres of human naevi. Nature 436: 720-724.

17. Quintanilla M (1986) Carcinogen-specific mutation and amplification of Ha-ras during mouse skin carcinogenesis. Nature 322:78-80.
18. Bennecke M (2010) Ink4a/Arf and oncogene-induced senescence prevent tumor progression during alternative colorectal tumorigenesis. Cancer Cell 18:135-146.

19. Courtois-Cox S (2006) A negative feedback signaling network underlies oncogene-induced senescence. Cancer Cell 10: 459-472.

20. Shamma A (2009) Rb Regulates DNA damage response and cellular senescence through E2F-dependent suppression of $\mathrm{N}$-ras isoprenylation. Cancer Cell 15: 255-269.

21. Young AP (2008) VHL loss actuates a HIF-independent senescence programme mediated by $\mathrm{Rb}$ and $\mathrm{p} 400$. Nat Cell Biol 10: 361-369.

22. Chen Z (2005)Crucial role of p53-dependent cellular senescence in suppression of Pten-deficient tumorigenesis. Nature 436: 725-730.

23. Gadhikar MA (2013) Chk1/2 inhibition overcomes the cisplatin resistance of head and neck cancer cells secondary to the loss of functional p53. Mol Cancer Ther 12: 1860-1873.

24. Bullock AN, Fersht AR (2001) Rescuing the function of mutant p53. Nat Rev Cancer 1: 68-76.

25. Bykov VJ (2002) Restoration of the tumor suppressor function to mutant p53 by a low-molecular-weight compound. Nat Med 8: 282-288.

26. Vassilev LT(2004) In vivo activation of the p53 pathway by small-molecule antagonists of MDM2. Science 303: 844-848.

27. Vazquez A (2008) The genetics of the p53 pathway, apoptosis and cancer therapy. Nat Rev Drug Discov7: 979-987.

28. Ventura A (2007) Restoration of $p 53$ function leads to tumour regression in vivo. Nature 445: 661-665.

29. Xue W (2007) Senescence and tumour clearance is triggered by p53 restoration in murine liver carcinomas. Nature 445: 656-660.

30. Salim KY (2016) COTI-2, a novel small molecule that is active against multiple human cancer cell lines in vitro and in vivo. Oncotarget.

31. Campaner S (2010) Cdk2 suppresses cellular senescence induced by the c-myc oncogene. Nat Cell Biol 12: 54-59.

32. Puyol M (2010) A synthetic lethal interaction between K-Ras oncogenes and Cdk4 unveils a therapeutic strategy for non-small cell lung carcinoma. Cancer Cell 18: 63-73.

33. Lin HK (2010) Skp2 targeting suppresses tumorigenesis by Arf-p53independent cellular senescence. Nature 464: 374-379.

34. Arndt GM, MacKenzie KL (2016) New prospects for targeting telomerase beyond the telomere. Nat Rev Cancer 16: 508-524.

35. Harley CB (2008) Telomerase and cancer therapeutics. Nat Rev Cancer 8:16779. 